\section{Ser trabalhadora remunerada ou dona de casa associa-se à qualidade de vida relacionada à saúde?}

\author{
Are there differences between paid women \\ workers and housewives in health-related quality \\ of life? \\ ¿Ser trabajadora remunerada o ama de casa se \\ asocia a la calidad de vida relacionada con la \\ salud?
}

\begin{abstract}
Resumo
Considerando que não há estudos brasileiros que avaliem a relação entre a inserção no mercado de trabalho e a qualidade de vida relacionada à saúde (QVRS) de mulheres, objetivou-se com a presente pesquisa verificar se existe associação entre ter ou não trabalho remunerado e a QVRS das mulheres, $e$, se o estrato socioeconômico modifica esta associação. Trata-se de estudo transversal de base populacional com amostra de 668 mulheres de 18 a 64 anos do Inquérito de Saúde de Campinas (ISACamp 2008/2009), utilizando-se o SF-36 para avaliar a QVRS. Ser dona de casa esteve associado à pior QVRS, sobretudo nos aspectos mentais, mas esta associação é modificada pelo nível socioeconômico. Nos segmentos de intermediária e baixa escolaridade e renda familiar, as donas de casa apresentaram pior QVRS que as trabalhadoras remuneradas, mas não houve diferença entre os dois segmentos nos estratos de alta escolaridade e renda. A pior QVRS das donas de casa enfatiza a importância de políticas públicas que visem a ampliar as oportunidades de inserção da mulher no mercado de trabalho e de acesso à educação.
\end{abstract}

Qualidade de Vida; Trabalho Feminino; Saúde da Mulher
Caroline Senicato 1

Margareth Guimarães Lima ${ }^{1}$

Marilisa Berti de Azevedo Barros 1 


\section{Introdução}

Embora as mulheres que são trabalhadoras remuneradas tendam a acumular tarefas da atividade profissional com as relacionadas ao cuidado da família e do domicílio, o que pode levar à fadiga, ao estresse e aos sintomas psíqui$\cos 1,2$, diversos estudos têm constatado melhores condições de saúde nas mulheres que exercem atividade remunerada em comparação às donas de casa 3,4,5,6. Segundo esses estudos, as trabalhadoras remuneradas apresentaram menores prevalências de doenças crônicas 3,4 , de limitações provocadas pelas doenças 4 , de transtornos mentais 5 , além de menores taxas de mortalidade 3,6 em relação às donas de casa.

Ainda que existam algumas evidências sobre o benefício do trabalho para a saúde das mulheres, são incipientes e com resultados controversos os estudos que analisaram a qualidade de vida relacionada à saúde (QVRS) 7,8,9,10,11,12,13. Para a avaliação da QVRS os estudos têm frequentemente utilizado o instrumento The Medical Outcomes Study 36-Item Short-Form (SF-36), que produz medidas do impacto das condições de saúde/doença sobre a funcionalidade do organismo, o bem-estar físico e mental, e o desempenho em atividades sociais 14,15,16. Estudos realizados na Polônia, utilizando o SF-36, verificaram que a inserção no mercado de trabalho apresentou associação positiva com a QVRS de mulheres nas análises bivariadas 7,8. Já estudo realizado na Austrália constatou que ser dona de casa foi associado com a pior saúde mental, avaliada pelo instrumento SF-36, mesmo após os ajustes por variáveis socioeconômicas e demográficas, comportamentos e uso de serviços 10. Segundo um estudo longitudinal realizado na Holanda com mulheres desempregadas, a recolocação no mercado de trabalho contribuiu significativamente para melhores médias de todas as escalas do SF36 , após os ajustes por idade, etnia, escolaridade, duração do benefício do seguro desemprego e condições de saúde prévias 11. Contudo, pesquisas realizadas no Irã não identificaram diferenças na QVRS entre trabalhadoras e donas de casa, após ajuste por idade, escolaridade e renda familiar 12,13. A ausência de associação entre QVRS e trabalho constatada por esses estudos não pode ser generalizada, levando-se em conta a especificidade da condição feminina nos diversos contextos culturais. Poucos estudos avaliaram a QVRS de mulheres segundo inserção no processo produtivo, entre os quais nenhum foi realizado no Brasil 7,8,9,10,11.

Uma revisão sistemática que objetivou verificar a existência de associação entre o bemestar de mulheres e a inserção no mercado de trabalho, concluiu que diferentes indicadores de saúde e o controle dos fatores de confusão nas análises multivariadas produzem resultados distintos sobre os efeitos do trabalho na saúde feminina 3. Em uma pesquisa realizada em Barcelona (Espanha), Artazcoz et al. ${ }^{4}$ verificaram melhor estado de saúde nas trabalhadoras em comparação às donas de casa, mas ressaltaram que as diferenças entre os dois segmentos dependem do nível de escolaridade das mulheres e das variáveis de ajustes utilizadas. No segmento de baixa escolaridade, as donas de casa apresentaram maiores prevalências de doenças crônicas e limitações delas decorrentes, em comparação às trabalhadoras remuneradas do mesmo segmento, mesmo após o ajuste pela idade e demanda familiar (número de filhos crianças e convivência com idosos). Contudo, essa associação não foi observada no segmento de mulheres com alta escolaridade 4 .

Considerando os achados na literatura de possível associação entre inserção no mercado de trabalho e condições de saúde das mulheres, que esta associação pode ser modificada pelo nível socioeconômico e que não há estudos brasileiros que abordem a relação entre inserção no mercado e a QVRS nas mulheres, os objetivos desta pesquisa foram verificar se existe associação em ser trabalhadora remunerada ou dona de casa com a QVRS e, avaliar se a escolaridade e a renda familiar per capita, como medidas do nível socioeconômico, modificam essa associação.

\section{Métodos}

Trata-se de um estudo transversal de base populacional, que utilizou dados do Inquérito de Saúde de Campinas (ISACamp 2008/2009), realizado em Campinas, São Paulo, Brasil, entre os anos de 2008 e 2009. Esse inquérito foi organizado pelo Centro Colaborador em Análise de Situação de Saúde, Departamento de Saúde Coletiva, Universidade Estadual de Campinas (Unicamp).

$\mathrm{O}$ inquérito visou a obter informações sobre condições de saúde, morbidades, comportamentos de saúde e uso de serviços de saúde, de três domínios de idade: adolescente (10 a 19 anos), adulto (20 a 59) e idoso (60 anos ou mais) O tamanho da amostra foi definido considerando a estimativa de uma proporção de 0,50 , com um erro máximo de 4 a 5 pontos percentuais, com intervalo de 95\% de confiança (IC95\%) e efeito de delineamento de 2 , resultando em 1.000 indivíduos para cada domínio de idade. Esperando 80\% de taxa de resposta, o tamanho da amostra foi corrigido para 1.250 indivíduos de cada domínio. 
O processo de amostragem do ISACamp 2008/2009 envolveu dois estágios: setores censitários e domicílios. No primeiro, foram sorteados 50 setores censitários da área urbana do Município de Campinas com probabilidade proporcional ao número de domicílios. Foi feito um sorteio sistemático, no qual os setores foram previamente ordenados pelo porcentual de chefes de família com nível universitário. No segundo estágio do processo amostral foram sorteados os domicílios nos 50 setores previamente selecionados. Para cada um dos domínios de idade foram sorteadas amostras independentes de domicílios. Com base na probabilidade dos moradores de cada grupo etário residir no domicílio, conforme dados do Censo Demográfico de 2000 (Instituto Brasileiro de Geografia e Estatística. http:/ /www. ibge.gov.br, acessado em 30/Mar/2015), foram sorteados 2.150, 700 e 3.900 domicílios, respectivamente para entrevistas com adolescentes, adultos e idosos. Foram entrevistados todos os residentes que fossem do domínio de idade sorteado para aquele domicílio. A descrição do plano de amostragem do inquérito está disponível em Alves 17.

As informações foram obtidas por meio de um questionário estruturado, previamente testado, aplicado por entrevistadores treinados e supervisionados. A entrevista foi feita diretamente com a pessoa selecionada. Foram coletadas informações demográficas, socioeconômicas, de condições de saúde, de comportamentos relacionados à saúde e de uso de serviços de saúde.

No presente estudo foram analisados somente os dados de adultos do sexo feminino na faixa etária de 18 a 64 anos de idade.

As variáveis analisadas neste estudo foram: - Qualidade de vida relacionada à saúde (QVRS): utilizou-se o questionário SF-36, versão 2. Esse instrumento avalia o impacto das condições de saúde/doença sobre a funcionalidade do organismo e o bem-estar. Cada escala do instrumento avalia o impacto que a doença causa em várias dimensões: física, social, emocional e mental. O SF-36 é composto de 36 questões, agrupadas em oito escalas: capacidade funcional, aspectos físicos (limitações decorrentes de problemas físicos), dor, saúde geral, vitalidade, aspectos emocionais (limitações decorrentes de problemas emocionais), aspectos sociais (atividades sociais e interações com membros familiares, amigos, vizinhos) e saúde mental; e dois componentes de medida que resumem estas escalas: o físico e o mental. O componente físico compreende as escalas de capacidade funcional, aspectos físicos e dor, e o componente mental é formado pelas escalas de aspectos sociais, emocionais e saúde mental. As escalas de vitalidade e de saúde geral fazem parte dos dois componentes. Em cada um dos oito domínios, os totais de escores foram convertidos para uma escala de 0 a 100. Zero corresponde ao pior estado de saúde e 100 ao melhor. Quanto aos dois componentes, as medidas foram padronizadas em relação à população americana (média de 50 e desvio padrão de 10) como recomendado por Ware et al. 14.

- Inserção no mercado de trabalho: categorizada em trabalhadoras remuneradas e donas de casa. Foram consideradas trabalhadoras remuneradas as mulheres que, no período em que a entrevista foi realizada, relataram ter algum tipo de atividade com remuneração. Foram também incluídas nessa categoria as mulheres temporariamente afastadas do trabalho por motivo de doença e as aposentadas que continuassem trabalhando de forma remunerada; foram consideradas donas de casa as demais mulheres que não exerciam atividade remunerada e referiram ser donas de casa. Mulheres desempregadas $(n=38)$, aposentadas $(n=101)$ e estudantes $(n=34)$ não foram incluídas na presente pesquisa.

- Variáveis demográficas e socioeconômicas: faixa etária (18 a 29, 30 a 39, 40 a 49 e 50 a 64 anos), escolaridade (avaliada em anos de estudos e categorizada em: 0 a 8, 9 a 12 e 13 ou mais), renda familiar mensal per capita (calculada em número de salários mínimos e categorizada em: $\leq 1$, > 1 a $<3$ e $\geq 3$ salários mínimos), situação conjugal (com companheiro, desquitada/separada/ divorciada/viúva e solteira) e número de filhos ( $0,1,2,3$ ou mais).

- Número de doenças crônicas: as referidas como tendo sido diagnosticadas por médico e levantadas por um checklist, que incluía: hipertensão arterial, diabetes, doença do coração, reumatismo/artrite/artrose, asma/bronquite/ enfisema, tendinite/LER/DORT e problema circulatório. Essa variável foi categorizada em: 0, 1, 2 e 3 ou mais doenças crônicas referidas.

Neste estudo, as variáveis dependentes foram as oito escalas e os dois componentes gerados pelo SF-36. A variável independente foi a inserção no mercado de trabalho (trabalhadoras remuneradas e donas de casa). O nível de escolaridade (anos de estudos) e a renda familiar per capita foram utilizados para a análise estratificada devido à heterogeneidade social que existe no interior dos dois grupos de mulheres: trabalhadoras remuneradas e donas de casa. As análises estratificadas verificaram se a associação entre trabalho remunerado e os escores das escalas e dos componentes da QVRS era diferente conforme o estrato de escolaridade ou de renda familiar per capita. As outras variáveis demográficas e socioeconômicas foram usadas para descrever as características dos dois segmentos de mulheres 
definidos pela inserção no mercado de trabalho. Idade, escolaridade, renda familiar per capita, situação conjugal, número de filhos e número de doenças crônicas foram utilizados para o controle de confundimento na análise ajustada.

Os dados oriundos do inquérito foram digitados em um banco no software EpiData versão 3.1 (Epidata Assoc., Odense, Dinamarca). As análises estatísticas foram realizadas com o software Stata versão 11.0 (StataCorp LP, College Station, Estados Unidos), módulo svy, que permite considerar os pesos amostrais e o efeito do delineamento do estudo. As associações entre as variáveis demográficas e socioeconômicas e a inserção no mercado de trabalho foram determinadas por meio do teste qui-quadrado, com nível de significância de 5\%. Modelos de regressão linear simples e múltipla (ajustados por idade, escolaridade, renda familiar per capita, situação conjugal, número de filhos e número de doenças crônicas) foram usados para verificar a associação das oito escalas e dos dois componentes do SF-36 com a inserção no mercado de trabalho. Os ajustes foram feitos porque há evidências científicas de que a QVRS diminui significativamente com o avanço da idade e o acúmulo de doenças, e é pior entre as mulheres com baixa escolaridade, renda e que não são mais casadas 7,8,18,19. A análise da comparação entre inserção no mercado de trabalho e QVRS também foi estratificada por escolaridade e renda familiar per capita. Foram realizadas as análises de resíduos e os resultados foram satisfatórios.

Esta pesquisa foi aprovada pelo Comitê de Ética da Faculdade de Ciências Médicas da Unicamp, em adendo ao parecer no 079/2007. Todas as mulheres participantes assinaram o Termo de Consentimento Livre e Esclarecido.

\section{Resultados}

A amostra estudada foi composta por $668 \mathrm{mu}$ lheres de 18 a 64 anos, com idade média de 38,4 (IC95\%: 37,4-39,4) anos; sendo de 37,1 (IC95\%: $36,0-38,2)$ anos a média de idade das trabalhadoras remuneradas e de 41,9 (IC95\%: 39,8-43,9) anos a das donas de casa. Da população adulta de mulheres do Município de Campinas, 31,5\% (IC95\%: 27,3-35,7) eram donas de casa. Já entre as trabalhadoras remuneradas, 2,6\% (IC95\%: 1,0$4,2)$ estavam afastadas por motivo de doença e 0,6\% (IC95\%: 0,3-1,0) era também aposentada.

As donas de casa eram em maior proporção mais velhas, com menor escolaridade, menor renda familiar per capita, casadas ou amasiadas e tinham dois ou mais filhos em relação às trabalhadoras remuneradas (Tabela 1).
Na Tabela 2, após os ajustes por idade, escolaridade, renda familiar per capita, situação conjugal, número de filhos e número de doenças crônicas, as donas de casa apresentaram menores médias dos escores em todas as escalas, com exceções nas escalas de capacidade funcional (limiar da significância estatística) e dor, e no componente físico, quando comparadas às mulheres que exercem algum trabalho remunerado. As maiores diferenças entre as médias dos escores foram verificadas nas escalas de saúde mental $(\beta=-7,8)$, vitalidade $(\beta=-6,6)$, aspectos físicos $(\beta=-6,4)$ e aspectos sociais $(\beta=-6,2)$. A diferença entre os dois segmentos de mulheres foi significativa no componente mental $(\beta=-4,1)$.

As diferenças na QVRS entre trabalhadoras remuneradas e donas de casa, estratificadas pelo nível de escolaridade, estão apresentadas na Tabela 3. Na análise ajustada por idade, renda familiar per capita, situação conjugal, número de filhos e número de doenças crônicas não houve diferença estatística na QVRS, avaliada pelas escalas do SF-36, entre trabalhadoras remuneradas e donas de casa que tinham 13 ou mais anos de escolaridade. No estrato intermediário, de 9 a 12 anos de escolaridade, as donas de casa apresentaram menores médias de escores nas escalas de vitalidade $(\beta=-14,0)$, saúde mental $(\beta=-12,7)$, saúde geral $(\beta=-7,0)$ e no componente mental $(\beta=-6,3)$. No estrato de menor nível de escolaridade (até 8 anos de estudos), as donas de casa, em comparação às trabalhadoras remuneradas, apresentaram menores escores em cinco das oito escalas do SF-36, não havendo diferença quanto às escalas de capacidade funcional, dor e de vitalidade. Diferença foi também constatada em relação ao componente mental. Na escala de saúde mental e no componente mental, as diferenças entre os escores de trabalhadoras e de donas de casa foram maiores no segmento de escolaridade intermediária do que no segmento de menor nível de escolaridade.

As diferenças na QVRS entre trabalhadoras remuneradas e donas de casa, estratificadas pelo nível de renda familiar per capita, estão apresentadas na Tabela 4. Na análise ajustada não houve diferença estatisticamente significativa na QVRS entre trabalhadoras remuneradas e donas de casa com renda familiar per capita maior ou igual a 3 salários mínimos. Já no estrato intermediário, com renda maior que 1 e menor que 3 salários mínimos, as donas de casa apresentaram menores médias de escores nas escalas de aspectos emocionais $(\beta=-8,6)$, aspectos sociais $(\beta=-7,4)$, saúde mental $(\beta=-7,4)$ e no componente mental $(\beta=-4,4)$. No estrato de menor renda familiar per capita (menor ou igual a 1 salário mínimo), as donas de casa, em comparação às trabalhadoras, 
Características demográficas e socioeconômicas e número de doenças crônicas de trabalhadoras remuneradas e donas de casa. ISACamp 2008/2009, Campinas, São Paulo, Brasil.

\begin{tabular}{|c|c|c|c|c|c|c|c|}
\hline \multirow[t]{3}{*}{ Variáveis } & \multicolumn{6}{|c|}{ Inserção no mercado de trabalho } & \multirow[t]{3}{*}{ Valor de $p$ * } \\
\hline & \multicolumn{2}{|c|}{ Total } & \multicolumn{2}{|c|}{ Trabalhadoras remuneradas } & \multicolumn{2}{|c|}{ Donas de casa } & \\
\hline & $\mathbf{n}$ & $\%$ & $\mathbf{n}$ & $\%$ & $\mathbf{n}$ & $\%$ & \\
\hline Faixa etária (anos) & & & & & & & $<0,0001$ \\
\hline $18-29$ & 171 & 27,9 & 127 & 29,6 & 44 & 24,4 & \\
\hline $30-39$ & 124 & 26,2 & 98 & 30,4 & 26 & 17,1 & \\
\hline $40-49$ & 118 & 24,3 & 81 & 24,4 & 37 & 24,1 & \\
\hline $50-64$ & 255 & 21,6 & 117 & 15,6 & 138 & 34,4 & \\
\hline Escolaridade (anos de estudo) & & & & & & & $<0,0001$ \\
\hline $0-8$ & 343 & 44,8 & 169 & 35,5 & 174 & 65,1 & \\
\hline $9-12$ & 173 & 27,9 & 122 & 29,3 & 51 & 24,6 & \\
\hline 13 ou + & 152 & 27,3 & 132 & 35,2 & 20 & 10,3 & \\
\hline Renda familiar per capita (salários mínimos) ** & & & & & & & 0,0064 \\
\hline$\leq 1$ & 289 & 43,2 & 165 & 37,7 & 124 & 55,2 & \\
\hline$>1 a<3$ & 255 & 36,6 & 165 & 38,9 & 90 & 31,8 & \\
\hline$\geq 3$ & 124 & 20,2 & 93 & 23,4 & 31 & 13,0 & \\
\hline Situação conjugal & & & & & & & $<0,0001$ \\
\hline Com companheiro & 418 & 64,6 & 229 & 57,4 & 189 & 80,3 & \\
\hline Desquitada/Separada/Divorciada/Viúva & 103 & 13,2 & 65 & 13,9 & 38 & 11,6 & \\
\hline Solteira & 147 & 22,2 & 129 & 29,7 & 18 & 8,1 & \\
\hline Número de filhos & & & & & & & $<0,0001$ \\
\hline 0 & 153 & 24,3 & 143 & 33,8 & 10 & 3,8 & \\
\hline 1 & 128 & 22,1 & 83 & 22,0 & 45 & 22,4 & \\
\hline 2 & 176 & 26,3 & 90 & 22,0 & 86 & 35,4 & \\
\hline 3 ou + & 211 & 27,3 & 107 & 22,2 & 104 & 38,4 & \\
\hline Número de doenças crônicas & & & & & & & 0,3898 \\
\hline 0 & 331 & 56,3 & 226 & 58,2 & 105 & 52,4 & \\
\hline 1 & 168 & 25,5 & 105 & 25,5 & 63 & 25,3 & \\
\hline 2 & 82 & 8,8 & 51 & 8,0 & 31 & 10,5 & \\
\hline $3 \mathrm{ou}+$ & 87 & 9,4 & 41 & 8,3 & 46 & 11,8 & \\
\hline Total & 668 & & 423 & & 245 & & \\
\hline
\end{tabular}

* Teste qui-quadrado;

** Salário mínimo vigente à época da pesquisa: janeiro a abril/2008: R\$ 415,00; maio/2008 a abril/2009: R\$ 450,00.

apresentaram menores escores em quatro das oito escalas do SF-36, havendo diferença quanto às escalas de aspectos físicos $(\beta=-6,3)$, saúde geral $(\beta=-9,5)$, vitalidade $(\beta=-9,8)$ e saúde mental $(\beta=-8,5)$. Diferença também foi constatada em relação ao componente mental $(\beta=-4,4)$.

\section{Discussão}

Embora muitas trabalhadoras remuneradas possam vir a acumular tarefas profissionais com o cuidado da família e da casa, foram as donas de casa que apresentaram pior QVRS. As donas de casa registraram os escores de seis escalas e do componente mental do SF-36 significativamente inferiores aos das trabalhadoras remuneradas, mesmo após ajuste por idade, escolaridade, renda familiar per capita, situação conjugal, número de filhos e número de doenças crônicas. Alguns estudos que utilizaram o mesmo instrumento em populações femininas da Holanda e da Austrália também constataram o benefício da ocupação na QVRS das mulheres, após os ajustes por variáveis socioeconômicas e demográficas, comportamentos e uso de serviços 10,11. Contudo, alguns 
Diferenças entre as médias dos escores ( $\beta$ ) das donas de casa em relação às trabalhadoras remuneradas nas análises brutas e ajustadas. ISACamp 2008/2009, Campinas, São Paulo, Brasil.

\begin{tabular}{|c|c|c|c|c|c|}
\hline \multirow[t]{2}{*}{ Escalas do SF-36 } & \multicolumn{3}{|c|}{ Médias e desvio padrão dos escores } & \multirow{2}{*}{$\begin{array}{l}\text { Análises brutas } \\
\beta^{\star \star \star}(\text { Valor de } p)\end{array}$} & \multirow{2}{*}{ 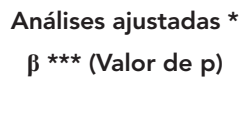 } \\
\hline & Total ** & $\begin{array}{l}\text { Trabalhadoras } \\
\text { remuneradas }\end{array}$ & Donas de casa & & \\
\hline Capacidade funcional & $92,6(91,0-94,2)$ & $94,8(93,2-96,5)$ & $89,7(87,2-92,2)$ & $-5,1(<0,001)$ & $-2,8(0,058)$ \\
\hline Aspectos físicos & $93,0(91,5-94,6)$ & $95,6(94,1-97,1)$ & $87,9(83,9-91,9)$ & $-7,7(0,001)$ & $-6,4(0,004)$ \\
\hline Dor & $84,8(82,2-87,4)$ & $85,9(83,3-88,5)$ & $82,7(77,9-87,5)$ & $-3,2(0,175)$ & $-1,2(0,589)$ \\
\hline Saúde geral & $79,0(76,5-81,5)$ & $82,3(80,2-84,3)$ & $73,6(68,8-78,4)$ & $-8,7(<0,001)$ & $-5,9(0,013)$ \\
\hline Vitalidade & $75,4(71,6-79,2)$ & $77,9(74,6-81,2)$ & $70,3(64,1-76,4)$ & $-7,6(0,001)$ & $-6,6(0,006)$ \\
\hline Aspectos emocionais & $93,3(91,8-94,7)$ & $96,0(94,8-97,2)$ & $89,9(86,2-93,7)$ & $-6,1(0,004)$ & $-5,7(0,006)$ \\
\hline Aspectos sociais & $89,9(87,9-91,9)$ & $93,2(91,4-95,0)$ & $86,1(81,8-90,4)$ & $-7,1(0,002)$ & $-6,2(0,003)$ \\
\hline Saúde mental & $77,8(74,3-81,2)$ & $80,6(77,7-83,5)$ & $71,7(65,9-77,4)$ & $-8,9(<0,001)$ & $-7,8(<0,001)$ \\
\hline Componente físico & $55,1(54,5-55,6)$ & $55,8(55,2-56,4)$ & $53,9(52,5-55,2)$ & $-1,9(0,015)$ & $-0,9(0,269)$ \\
\hline Componente mental & $52,7(51,2-54,3)$ & $54,3(53,1-55,6)$ & $50,0(47,3-52,8)$ & $-4,3(<0,001)$ & $-4,1(<0,001)$ \\
\hline
\end{tabular}

SF-36: The Medical Outcomes Study 36-Item Short-Form.

* Ajustadas por idade, escolaridade, renda familiar per capita, situação conjugal, número de filhos e número de doenças crônicas;

** Médias dos escores de todas as mulheres, das trabalhadoras remuneradas e das donas de casa;

*** Betacoeficientes, resultados de regressão linear, verificados pelo teste t de Student. Diferenças entre as médias dos escores das escalas das donas de casa e das trabalhadoras remuneradas.

estudos realizados no Irã comparando a QVRS entre trabalhadoras remuneradas e donas de casa não encontraram diferenças entre estes dois segmentos, após os ajustes por variáveis socioeconômicas 12,13. Mas, é preciso considerar que as divergências dos resultados podem decorrer de particularidades culturais relacionadas ao papel da mulher na sociedade e ao grau de valorização de sua inserção no processo de trabalho.

Uma revisão sistemática de estudos longitudinais sobre o efeito do emprego no bem-estar das mulheres também constatou que o trabalho remunerado tem efeitos benéficos sobre a saúde feminina. Os autores detectaram menores prevalências de sofrimento psíquico, de doenças crônicas, de riscos cardiovasculares, e até mesmo menor taxa de mortalidade entre as mulheres que tinham trabalho remunerado ${ }^{3}$. Em outra revisão sistemática recentemente publicada, mas com adultos de ambos os sexos, os autores encontraram forte evidência do efeito protetor do emprego sobre a depressão e a saúde mental em geral, mas não para a saúde física e a mortalidade, concluindo que há insuficiência de estudos para afirmativas consistentes 20 .

Ainda que exista associação entre inserção no mercado de trabalho e QVRS, esta pesquisa destaca que pertencer a determinado estrato socioeconômico modifica esta associação. Nas mulheres com alto grau de escolaridade não existem dife- renças nos escores da QVRS entre aquelas com ocupação remunerada e as donas de casa. Diferentemente do observado nos níveis de escolaridade intermediário e baixo, em que as donas de casa registraram qualidade de vida relacionada à saúde consistentemente pior em comparação às trabalhadoras remuneradas. Resultados similares foram encontrados ao estratificar pela renda familiar per capita. Em um estudo realizado na Espanha, Artazcoz et at. 4 também já haviam constatado que o nível socioeconômico atuava como um modificador de efeito na relação entre inserção no mercado de trabalho e condições de saúde de mulheres adultas.

É plausível que nos segmentos com piores condições socioeconômicas (baixa escolaridade e renda familiar per capita) as donas de casa tenham menor autonomia e poucas perspectivas de desenvolvimento e satisfação pessoal, comparadas às mulheres de mesmo estrato social, mas que têm um trabalho remunerado. Um estudo transversal realizado no Japão mostra a importância da renda familiar para a qualidade de vida relacionada à saúde das donas de casa que, por não receberem salário, têm uma relação de dependência dos outros membros da família. Os autores verificaram a associação entre baixa renda familiar e menor pontuação na escala de saúde geral, do SF-36, nas donas de casa 21 . Um estudo longitudinal mostrou que o aumento da 
Diferenças entre as médias dos escores $(\beta)$ das donas de casa em relação às trabalhadoras remuneradas em três estratos de escolaridade nas análises brutas e ajustadas. ISACamp 2008/2009, Campinas, São Paulo, Brasil.

\begin{tabular}{|c|c|c|c|c|c|c|}
\hline \multirow[t]{5}{*}{ Escalas do SF-36 } & \multicolumn{6}{|c|}{ Análises brutas } \\
\hline & \multicolumn{6}{|c|}{ Escolaridade (anos de estudo) } \\
\hline & \multicolumn{2}{|c|}{13 ou +} & \multicolumn{2}{|c|}{$9-12$} & \multicolumn{2}{|c|}{ Até 8} \\
\hline & $\begin{array}{c}\text { Trabalhadoras } \\
\text { remuneradas } \\
(n=132)\end{array}$ & $\begin{array}{l}\text { Donas de casa } \\
\qquad(n=20)\end{array}$ & $\begin{array}{c}\text { Trabalhadoras } \\
\text { remuneradas } \\
(n=122)\end{array}$ & $\begin{array}{l}\text { Donas de casa } \\
\qquad(n=51)\end{array}$ & $\begin{array}{c}\text { Trabalhadoras } \\
\text { remuneradas } \\
(n=169)\end{array}$ & $\begin{array}{c}\text { Donas de casa } \\
\quad(n=174)\end{array}$ \\
\hline & Média * & $\beta$ ** (valor de $p)$ & Média * & $\beta * \star($ valor de $p)$ & Média * & $\beta$ ** (valor de $p)$ \\
\hline Capacidade funcional & 97,2 & $-2,3(0,481)$ & 94,9 & $1,7(0,366)$ & 92,4 & $-4,9(0,055)$ \\
\hline Aspectos físicos & 97,6 & $-1,4(0,613)$ & 97,2 & $-2,9(0,321)$ & 92,3 & $-8,1(0,016)$ \\
\hline Dor & 89,4 & $-2,5(0,660)$ & 86,8 & $-2,2(0,635)$ & 81,7 & $-0,4(0,896)$ \\
\hline Saúde geral & 85,6 & $3,0(0,399)$ & 86,2 & $-6,6(0,016)$ & 75,8 & $-6,8(0,042)$ \\
\hline Vitalidade & 81,2 & $-0,8(0,885)$ & 79,3 & $-11,2(0,001)$ & 73,5 & $-4,0(0,233)$ \\
\hline Aspectos emocionais & 97,5 & $0,7(0,702)$ & 97,1 & $-5,9(0,057)$ & 93,6 & $-5,5(0,063)$ \\
\hline Aspectos sociais & 95,7 & $0,3(0,904)$ & 93,7 & $-3,6(0,292)$ & 90,1 & $-7,2(0,029)$ \\
\hline Saúde mental & 83,5 & $-0,9(0,750)$ & 82,2 & $-11,2(0,006)$ & 76,4 & $-6,2(0,053)$ \\
\hline Componente físico & 56,9 & $-0,5(0,737)$ & 56,3 & $-0,07(0,955)$ & 54,2 & $-1,6(0,132)$ \\
\hline Componente mental & 55,6 & $0,2(0,886)$ & 55,1 & $-5,5(0,003)$ & 52,4 & $-3,2(0,059)$ \\
\hline \multirow[t]{5}{*}{ Escalas do SF-36 } & \multicolumn{6}{|c|}{ 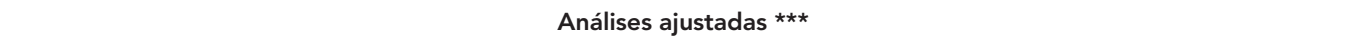 } \\
\hline & \multicolumn{6}{|c|}{ Escolaridade (anos de estudo) } \\
\hline & \multicolumn{2}{|c|}{13 ou +} & \multicolumn{2}{|c|}{$9-12$} & \multicolumn{2}{|c|}{ Até 8} \\
\hline & $\begin{array}{l}\text { Trabalhadoras } \\
\text { remuneradas } \\
(n=132)\end{array}$ & $\begin{array}{l}\text { Donas de casa } \\
\qquad(n=20)\end{array}$ & $\begin{array}{c}\text { Trabalhadoras } \\
\text { remuneradas } \\
(n=122)\end{array}$ & $\begin{array}{l}\text { Donas de casa } \\
\qquad(n=51)\end{array}$ & $\begin{array}{c}\text { Trabalhadoras } \\
\text { remuneradas } \\
(n=169)\end{array}$ & $\begin{array}{c}\text { Donas de casa } \\
\qquad(n=174)\end{array}$ \\
\hline & Média * & $\beta$ ** (valor de $p)$ & Média * & $\beta$ ** (valor de $p)$ & Média * & $\beta$ ** (valor de $p)$ \\
\hline Capacidade funcional & 97,2 & $-2,1(0,499)$ & 94,9 & $0,1(0,961)$ & 92,4 & $-4,0(0,119)$ \\
\hline Aspectos físicos & 97,6 & $-1,9(0,435)$ & 97,2 & $-2,5(0,530)$ & 92,3 & $-9,0(0,005)$ \\
\hline Dor & 89,4 & $-0,6(0,903)$ & 86,8 & $-1,4(0,792)$ & 81,7 & $-0,9(0,729)$ \\
\hline Saúde geral & 85,6 & $5,6(0,121)$ & 86,2 & $-7,0(0,010)$ & 75,8 & $-8,3(0,016)$ \\
\hline Vitalidade & 81,2 & $0,8(0,866)$ & 79,3 & $-14,0(<0,001)$ & 73,5 & $-5,7(0,074)$ \\
\hline Aspectos emocionais & 97,5 & $0,8(0,785)$ & 97,1 & $-4,6(0,160)$ & 93,6 & $-7,7(0,015)$ \\
\hline Aspectos sociais & 95,7 & $1,4(0,719)$ & 93,7 & $-4,4(0,241)$ & 90,1 & $-9,2(0,004)$ \\
\hline Saúde mental & 83,5 & $0,8(0,834)$ & 82,2 & $-12,7(0,005)$ & 76,4 & $-7,9(0,007)$ \\
\hline Componente físico & 56,9 & $-0,2(0,871)$ & 56,3 & $0,4(0,804)$ & 54,2 & $-1,5(0,171)$ \\
\hline Componente mental & 55,6 & $0,9(0,652)$ & 55,1 & $-6,3(0,003)$ & 52,4 & $-4,5(0,007)$ \\
\hline
\end{tabular}

SF-36: The Medical Outcomes Study 36-Item Short-Form.

* Médias dos escores;

** Betacoeficientes, resultados de regressão linear, verificados pelo teste t de Student. Diferenças entre as médias dos escores das escalas das donas de casa e das trabalhadoras remuneradas:

*** Ajustadas por idade, renda familiar per capita, situação conjugal, número de filhos e número de doenças crônicas.

renda das mulheres que trabalhavam fora, e da contribuição deste ganho na renda familiar, repercutiu significativa e favoravelmente na felicidade conjugal e no bem-estar 22 . Mesmo que as trabalhadoras com baixa escolaridade recebam baixos salários, a atividade ocupacional propicia uma renda pessoal, uma melhora da renda familiar, além de apoio social e oportunidades para o aumento da autoestima e confiança e o desenvolvimento de habilidades para lidar com situações de estresse e para a construção de redes sociais ${ }^{3,23}$. Em um estudo desenvolvido na Nova 
Tabela 4

Diferenças entre as médias dos escores $(\beta)$ das donas de casa em relação às trabalhadoras remuneradas em três estratos de renda familiar per capita nas análises brutas e ajustadas. ISACamp 2008/2009, Campinas, São Paulo, Brasil.

\begin{tabular}{|c|c|c|c|c|c|c|}
\hline \multirow[t]{5}{*}{ Escalas do SF-36 } & \multicolumn{6}{|c|}{ Análises brutas } \\
\hline & \multicolumn{6}{|c|}{ Salários mínimos * } \\
\hline & \multicolumn{2}{|c|}{$\geq 3$} & \multicolumn{2}{|c|}{$>1 \mathrm{a}<3$} & \multicolumn{2}{|c|}{$\leq 1$} \\
\hline & $\begin{array}{l}\text { Trabalhadoras } \\
\text { remuneradas } \\
\qquad(n=93)\end{array}$ & $\begin{array}{l}\text { Donas de casa } \\
\qquad(n=31)\end{array}$ & $\begin{array}{c}\text { Trabalhadoras } \\
\text { remuneradas } \\
(n=165)\end{array}$ & $\begin{array}{l}\text { Donas de casa } \\
\qquad(n=90)\end{array}$ & $\begin{array}{c}\text { Trabalhadoras } \\
\text { remuneradas } \\
(n=165)\end{array}$ & $\begin{array}{l}\text { Donas de casa } \\
\qquad(n=124)\end{array}$ \\
\hline & Média ** & $\beta^{\star \star \star}($ valor de $\mathbf{p})$ & Média ** & $\beta^{\star \star \star}($ valor de $\mathbf{p})$ & Média ** & $\begin{array}{c}\beta * \star \star \text { (valor } \\
\text { de } \mathbf{p})\end{array}$ \\
\hline Capacidade funcional & 96,6 & $-4,1(0,039)$ & 94,7 & $-6,3(0,001)$ & 93,9 & $-4,1(0,073)$ \\
\hline Aspectos físicos & 96,7 & $-4,0(0,315)$ & 96,1 & $-10,7(0,011)$ & 94,5 & $-6,2(0,023)$ \\
\hline Dor & 87,0 & $-2,7(0,370)$ & 86,7 & $-6,7(0,056)$ & 84,4 & $-0,6(0,884)$ \\
\hline Saúde geral & 86,6 & $-2,2(0,628)$ & 82,8 & $-9,1(0,004)$ & 79,0 & $-8,1(0,021)$ \\
\hline Vitalidade & 81,7 & $-5,4(0,293)$ & 77,8 & $-6,7(0,049)$ & 75,7 & $-7,3(0,025)$ \\
\hline Aspectos emocionais & 96,3 & $-2,2(0,348)$ & 97,1 & $-10,9(0,002)$ & 94,7 & $-3,5(0,265)$ \\
\hline Aspectos sociais & 94,8 & $-1,4(0,608)$ & 94,2 & $-11,8(0,003)$ & 91,1 & $-4,6(0,181)$ \\
\hline Saúde mental & 85,2 & $-8,7(0,010)$ & 80,6 & $-10,1(0,003)$ & 77,8 & $-6,5(0,041)$ \\
\hline Componente físico & 56,3 & $-0,7(0,599)$ & 55,9 & $-2,5(0,035)$ & 55,3 & $-1,6(0,149)$ \\
\hline Componente mental & 56,0 & $-2,9(0,045)$ & 54,6 & $-5,6(0,001)$ & 53,0 & $-3,1(0,081)$ \\
\hline \multirow[t]{5}{*}{ Escalas do SF-36 } & \multicolumn{6}{|c|}{ Análises ajustadas \# } \\
\hline & \multicolumn{6}{|c|}{ Salários mínimos * } \\
\hline & \multicolumn{2}{|c|}{$\geq 3$} & \multicolumn{2}{|c|}{$>1 \mathrm{a}<3$} & \multicolumn{2}{|c|}{$\leq 1$} \\
\hline & $\begin{array}{l}\text { Trabalhadoras } \\
\text { remuneradas } \\
\qquad(n=93)\end{array}$ & $\begin{array}{l}\text { Donas de casa } \\
\qquad(n=31)\end{array}$ & $\begin{array}{l}\text { Trabalhadoras } \\
\text { remuneradas } \\
(n=165)\end{array}$ & $\begin{array}{l}\text { Donas de casa } \\
\qquad(n=90)\end{array}$ & $\begin{array}{c}\text { Trabalhadoras } \\
\text { remuneradas } \\
(n=165)\end{array}$ & $\begin{array}{l}\text { Donas de casa } \\
\qquad(n=124)\end{array}$ \\
\hline & Média ** & $\beta^{\star \star \star}($ valor de $\mathbf{p})$ & Média ** & $\beta^{\star \star \star}($ valor de $\mathbf{p})$ & Média ** & $\begin{array}{c}\beta^{\star \star \star} \text { (valor } \\
\text { de } \mathbf{p})\end{array}$ \\
\hline Capacidade funcional & 96,6 & $-2,6(0,215)$ & 94,7 & $-1,7(0,376)$ & 93,9 & $-2,2(0,290)$ \\
\hline Aspectos físicos & 96,7 & $-6,5(0,342)$ & 96,1 & $-6,6(0,096)$ & 94,5 & $-6,3(0,015)$ \\
\hline Dor & 87,0 & $-7,4(0,277)$ & 86,7 & $0,7(0,861)$ & 84,4 & $-2,4(0,432)$ \\
\hline Saúde geral & 86,6 & $3,5(0,326)$ & 82,8 & $-3,0(0,242)$ & 79,0 & $-9,5(0,011)$ \\
\hline Vitalidade & 81,7 & $-0,8(0,154)$ & 77,8 & $-2,6(0,482)$ & 75,7 & $-9,8(0,004)$ \\
\hline Aspectos emocionais & 96,3 & $0,4(0,889)$ & 97,1 & $-8,6(0,019)$ & 94,7 & $-4,6(0,181)$ \\
\hline Aspectos sociais & 94,8 & $1,1(0,699)$ & 94,2 & $-7,4(0,029)$ & 91,1 & $-6,3(0,064)$ \\
\hline Saúde mental & 85,2 & $-5,6(0,135)$ & 80,6 & $-7,4(0,033)$ & 77,8 & $-8,5(0,002)$ \\
\hline Componente físico & 56,3 & $1,1(0,325)$ & 55,9 & $0,1(0,893)$ & 55,3 & $-1,4(0,184)$ \\
\hline Componente mental & 56,0 & $-1,3(0,404)$ & 54,6 & $-4,4(0,016)$ & 53,0 & $-4,4(0,010)$ \\
\hline
\end{tabular}

SF-36: The Medical Outcomes Study 36-Item Short-Form.

* Salário mínimo vigente à época da pesquisa: janeiro a abril/2008: R\$415,00; maio/2008 a abril/2009: R\$ 450,00;

** Médias dos escores;

*** Betacoeficientes, resultados de regressão linear, verificados pelo teste t de Student. Diferenças entre as médias dos escores das escalas das donas de casa e das trabalhadoras remuneradas;

\# Ajustadas por idade, escolaridade, situação conjugal, número de filhos e número de doenças crônicas. 
Zelândia e nos Estados Unidos, os autores constataram que as trabalhadoras remuneradas consideraram as amizades no local de trabalho como um suporte social e emocional nos momentos de estresse, diferentemente dos homens 24 .

Verificou-se nos níveis intermediário e baixo de escolaridade (bem como nos níveis intermediário e baixo de renda familiar per capita) que as donas de casa apresentaram pior QVRS em várias escalas, principalmente naquelas referentes à saúde mental e, consequentemente, no componente mental. Foram verificadas maiores diferenças de escores nas escalas de aspectos emocionais (que são as limitações devido às questões emocionais), aspectos sociais (atividades sociais e interações com membros familiares e amigos) e saúde mental (relacionada ao humor). Um estudo desenvolvido em um município no Nordeste do Brasil encontrou maior prevalência de transtorno mental comum entre as donas de casa, ao compará-las com trabalhadoras remuneradas 5. Em inquérito telefônico norte-americano, Lennon \& Rosenfield 25 constataram que a rotina das donas de casa com as tarefas domésticas diárias esteve associada à presença de sintomas depressivos. Além da rotina, a sobrecarga de trabalho doméstico, como lavar, passar, limpar e cozinhar, ponderada pelo número de moradores no domicílio, foi apontada por estudo transversal, realizado no Brasil, como associada positivamente à prevalência de transtornos mentais comuns, sendo que o percentual de alta sobrecarga doméstica foi maior entre as mulheres que ficavam em casa 26. É possível que a fragmentação, a rotina, a monotonia, poucas redes sociais e o baixo status do trabalho doméstico 23,24,25 aliados à pior condição socioeconômica colaborem para a pior QVRS entre as donas de casa. A inserção no mercado de trabalho entre as socioeconomicamente mais desfavorecidas, que contribui para o aumento da renda, das interações sociais e da sensação de inclusão na sociedade 6 , constituiria um mecanismo para alimentar aspirações e perspectivas de vida e, consequentemente, proporcionar maior bem-estar físico e mental.

Por outro lado, o fato de não ter sido observada diferença na QVRS entre trabalhadoras remuneradas e donas de casa no segmento de mulheres com alta escolaridade e renda, pode ser consequência das condições propiciadas pela melhor situação socioeconômica da mulher (escolaridade e renda familiar per capita). Donas de casa com elevado nível de estudo podem dispor de mais recursos e apoio para o cuidado da casa e dos filhos, amenizando a rotina e a monotonia das funções domésticas. A alta escolaridade possibilitaria à dona de casa conciliar o papel dentro do lar com outras atividades físicas e culturais que poderiam colaborar com a sua saúde e qualidade de vida, anulando a desigualdade na QVRS segundo a inserção no mercado de trabalho.

Embora não seja o foco do presente estudo, os resultados permitem constatar que tanto entre as mulheres que têm trabalho remunerado como nas donas de casa, quanto menor o nível de escolaridade e de renda familiar per capita, mais baixos são os escores das escalas do SF-36. Esse achado é consistente com pesquisas que analisaram mulheres utilizando o SF-36, realizadas em diferentes países como Espanha, Grécia, Polônia, Japão e Austrália, nos quais verificou-se a associação positiva entre nível socioeconômico, avaliado pela escolaridade ou pela renda, e a QVRS 7,10,19,21,27,28. A escolaridade está associada ao status ocupacional, visto que melhor nível educacional propicia maior oportunidade de exercer ocupações mais qualificadas, com melhor remuneração e mais autonomia e prestígio 29 . Além disso, o nível educacional contribui para a aquisição de informações e adoção de comportamentos saudáveis. Pior condição de saúde, avaliada por meio de vários indicadores nas mulheres com menor nível de escolaridade, já havia sido constatada previamente no mesmo município da presente pesquisa 30 .

Em decorrência do corte transversal do estudo não é possível afirmar que a melhor QVRS das mulheres com trabalho remunerado (em comparação às donas de casa), entre as pertencentes aos estratos socioeconômicos menos favorecidos, é proporcionada pelo trabalho ou se a associação é consequência do "efeito do trabalhador sadio", ou seja, as mulheres com pior estado de saúde teriam menor chance de inserção e permanência no mercado de trabalho. $\mathrm{O}$ "efeito do trabalhador sadio" deve, portanto, ser considerado, mas revisões sistemáticas de estudos longitudinais têm evidenciado efeitos benéficos do trabalho à saúde de homens e mulheres 3,20.

Não existem muitos estudos no Brasil analisando as condições de saúde das mulheres segundo a inserção no mercado de trabalho 4,31, sendo esta a primeira pesquisa brasileira de base populacional a comparar, entre trabalhadoras remuneradas e donas de casa, os domínios e componentes de saúde avaliados com o uso do SF-36. Espera-se que os resultados contribuam para instigar o desenvolvimento de mais estudos nesse tema, para ampliar a compreensão das condições que afetam a saúde das mulheres e possibilitar a avaliação das diferenças nas associações entre trabalho feminino e saúde em diferentes contextos culturais e segmentos sociais. Destaca-se o importante achado desta pesquisa quanto à identificação do nível socioeconômico 
como modificador do efeito da inserção no mercado de trabalho na QVRS feminina.

Um estudo realizado em vinte e oito países destacou que as diferenças no bem-estar feminino entre eles, além de envolver aspectos culturais, podem depender do contexto de vida de cada local, como o Produto Interno Bruto (PIB), os gastos sociais, a participação feminina na força de trabalho, a igualdade de gênero e o suporte público no cuidado com os filhos e com a família, como a presença de creches ${ }^{32}$. O emprego pode ser benéfico à saúde feminina se trabalhar fora estiver em sintonia com as convenções de gênero do país, o que envolve menor pressão sobre as mulheres por elas trabalharem. Portanto, segundo Tesch-Romer et al. 33, o bem-estar feminino está positivamente associado à participação da mulher no mercado de trabalho em países que apoiam a igualdade de gênero.

\section{Colaboradores}

C. Senicato realizou a proposta do artigo, a revisão da literatura, a análise dos dados e redação do artigo. M. G. Lima participou da análise dos dados e da redação do artigo. M. B. A. Barros orientou a proposta do artigo, a análise dos dados e participou da redação do manuscrito.

\section{Referências}

1. Macran S. Role enhancementor role overload? A review of research on the health consequence of women's domestic and paid work. London: Centre for Population Studies, London School of Hygiene and Tropical Medicine; 1993.

2. Santana VS, Loomis DP, Newman B. Housework, paid work and psychiatric symptoms. Rev Saúde Pública 2001; 35:16-22.
Os resultados deste estudo evidenciam a importância de políticas sociais voltadas a ampliar o acesso à educação feminina, a criar meios que possibilitem maior inserção da mulher no mercado de trabalho sem desvalorizar a relevância do trabalho doméstico. Além disso, a saúde mental das donas de casa, especialmente as de menor nível socioeconômico, deve ser alvo de atenção dos serviços de saúde na perspectiva de propiciar a detecção dos comprometimentos e de oferecer as melhores estratégias de controle.

\section{Agradecimentos}

À Fundação de Amparo à Pesquisa do Estado de São Paulo (FAPESP) pela bolsa de doutorado de C. Senicato (processo no 2012/07039-7); ao Conselho Nacional de Desenvolvimento Científico e Tecnológico (CNPq, processo no 409747 /2006-8) pelo financiamento da pesquisa e pela bolsa de produtividade de M. B. A. Barros; ao Ministério da Saúde e à Secretaria de Saúde de Campinas pelo apoio financeiro para a realização do inquérito (Convênio Unicamp/Funcamp/SMS no 4300).

3. Klumb PL, Lampert T. Women, work, and well-being 1950-2000: a review and methodological critique. Soc Sci Med 2004; 58:1007-24.

4. Artazcoz L, Borrell C, Benach J, Cortès I, Rohlfs I. Women, family demands and health: the importance of employment status and socio-economic position. Soc Sci Med 2004; 59:263-74. 
5. Araújo TM, Almeida MMG, Santana CC, Araújo EM, Pinho PS. Transtornos mentais comuns em mulheres: estudo comparativo entre donas-decasa e trabalhadoras. Rev Enferm UERJ 2006; 14: 260-9.

6. Weatherall R, Joshi H, Macran S. Double burden or double blessing? Employment, motherhood and mortality in the longitudinal study of England and Wales. Soc Sci Med 1994; 38:285-97.

7. Wieder-Huszla S, Szkup M, Jurczak A, Samochowiec A, Samochowiec J, Stanislawska M, et al. Effects of socio-demographic, personality and medical factors on quality of life of postmenopausal women. Int J Environ Res Public Health 2014; 11:6692-708.

8. Zolnierczuk-Kieliszek D, Kulik TB, Janiszewska $\mathrm{M}$, Stefanowicz A. Influence of sociodemographic factors on quality of life in women living in Lublin Province in Poland. Prz Menopauzalny 2014; 18:13-7.

9. D'Souza MS, Karkada SN, Somayaji G. Factors associated with health-related quality of life among Indian women in mining and agriculture. Health Qual Life Outcomes 2013; 11:9.

10. Outram S, Mishra GD, Schofield MJ. Sociodemographic and health related factors associated with poor mental health in midlife Australian women. Women Health 2004; 39:97-115.

11. Schuring M, Mackenbach J, Voorham T, Burdorf A. The effect of re-employment on perceived health. J Epidemiol Community Health 2011; 65:639-44.

12. Kerman Saravi F, Navidian A, Rigi SN, Montazeri A. Comparing health-related quality of life of employed women and housewives: a cross sectional study from southeast Iran. BMC Women Health 2012; 12:41.

13. Ahmad-Nia S. Women's work and health in Iran: a comparison of working and non-working mothers. Soc Sci Med 2002; 54:753-65.

14. Ware Jr. JE, Kosinski M, Bjorner JB, Turner-Bowker DM, Gandek B, Maruish ME. User's manual for the SF-36v2@ health survey. 2nd Ed. Lincoln: Quality Metric Inc.; 2007.

15. Huang IC, Wu AW, Frangakis C. Do the SF-36 and WHOQOL-BREF measure the same constructs? Evidence from the Taiwan population. Qual Life Res 2006; 15:15-24.

16. McDowell I. Measuring health: a guide to rating scales and questionnaires. 3rd Ed. New York: Oxford University Press; 2006.

17. Alves MCGP. ISA-Campinas 2008-2009: plano de amostragem. http://www.fcm.unicamp.br/fcm/ sites/default/files/plano_de_amostragem.pdf (acessado em 30/Mar/2015).

18. Lima MG, Barros MBA, César CLG, Goldbaum M, Carandina L, Ciconelli RM. Health related quality of life among the elderly: a population-based study using SF-36 survey. Cad Saúde Pública 2009; 25:2159-67.
19. Regidor E, Barrio G, de la Fuente L, Domingo A, Rodriguez C, Alonso J. Association between educational level and health-related quality of life in Spanish adults. J Epidemiol Community Health 1999; 53:75-82.

20. Noordt M, Ijzelenberg H, Droomers M, Proper KI. Health effects of employment: a systematic review of prospective studies. Occup Environ Med 2014; 71:730-6.

21. Yamazaki S, Fukuhara S, Suzukamo Y. Household income is strongly associated with health-related quality of life among Japanese men but not women. Public Health 2005; 119:561-7.

22. Rogers SJ, De Boer DD. Changes in wives' income: effects on marital happiness, psychological wellbeing, and the risk of divorce. J Marriage Fam 2001; 63:458-72.

23. Brown GW, Harris T. Social origins of depression: a study of psychiatric disorder in women. London: Tavistock; 1978.

24. Morrison RL. Are women tending and befriending in the workplace? Gender differences in the relationship between workplace friendships and organizational outcomes. Sex Roles 2009; 60:1-13.

25. Lennon MC, Rosenfield S. Women and mental health: the interaction of job and family conditions. J Health Soc Behav 1992; 33:316-27.

26. Pinho PS, Araújo TM. Associação entre sobrecarga doméstica e transtornos mentais comuns em mulheres. Rev Bras Epidemiol 2012; 15:560-72.

27. Guallar-Castillón P, Sendino AR, Banegas JR, Lópes-García E, Rodríguez-Artalejo F. Differences in quality of life between women and men in the older population of Spain. Soc Sci Med 2005; 60:1229-40.

28. Pappa E, Kontodilmopoulos N, Papadopoulos AA, Niakas D. Assessing the socio-economic and demographic impact on health-realted quality of life: evidence from Greece. Int J Public Health 2009; 54:241-9.

29. Ferraro AR. Escolarização no Brasil: articulando as perspectivas de gênero, raça e classe social. Educação e Pesquisa 2010; 36:505-26.

30. Senicato C, Barros MBA. Social inequality in health among women in Campinas, São Paulo State, Brazil. Cad Saúde Pública 2012; 28:1903-14.

31. Ludermir AB. Inserção produtiva, gênero e saúde mental. Cad Saúde Pública 2000; 16:647-59.

32. Treas J, van der Lippe T, Chloe Tai T, The happy homemaker? Married women's well-being in cross-national perspective. Soc Forces 2011; 90:111-32.

33. Tesch-Romer C, Motel-Klingebiel A, Tomasik MJ. Gender differences in subjective well-being: comparing societies with respect to gender equality. Soc Indic Res 2008; 85:329-49. 


\section{Abstract}

Considering the lack of Brazilian studies on the relationship between participation in the labor market and health-related quality of life (HRQL) in women, the objectives were to verify whether there is an association between paid work versus no paid work and $H R Q L$ in women, and whether socioeconomic status modifies this association. This was a populationbased cross-sectional study with a sample of $668 \mathrm{wom}$ en 18 to 64 years of age from the Campinas Health Survey (ISACamp 2008/2009), using SF-36 to assess HRQL. Being a housewife was associated with worse HRQL, especially in mental domains, but this association was modified by socioeconomic status. In the middle and low schooling and family income strata, housewives showed worse HRQL than paid women workers, but there was no difference between the two groups in the high schooling and high income strata. Housewives worse HRQL emphasizes the importance of public policies to expand opportunities for women's participation in the labor market and access to education.

Quality of Life; Working Women; Women's Health

\section{Resumen}

Considerando que no existen estudios brasileños que evalúen la relación entre inserción en el mercado de trabajo y la calidad de vida relacionada con la salud (QVRS) de mujeres, el objetivo de la presente investigación fue verificar si existe asociación entre tener o no trabajo remunerado y la QVRS de las mujeres, y si el estrato socioeconómico modifica esta asociación. Se trata de un estudio transversal de base poblacional con una muestra de 668 mujeres de 18 a 64 años de la Encuesta de Salud de Campinas (ISACamp 2008/2009), siendo utilizado el SF-36 para evaluar la QVRS. Ser ama de casa se asoció con la peor QVRS sobre todo en los aspectos mentales, pero esta asociación está modificada por el nivel socioeconómico. En los segmentos intermedios y baja escolaridad y renta familiar, las amas de casa presentaron un peor QVRS que las trabajadoras remuneradas, pero no hubo diferencia entre los dos segmentos en los estratos de alta escolaridad y renta. La peor QVRS de las amas de casa enfatiza la importancia de políticas públicas que tengan por objetivo ampliar las oportunidades de inserción de la mujer en el mercado de trabajo y de acceso a la educación.

Calidad de Vida; Trabajo de Mujeres;

Salud de la Mujer
Recebido em 31/Mai/2015

Versão final reapresentada em 14/Nov/2015 Aprovado em 18/Fev/2016 